\title{
GLOBAL MAGNETOFLUIDOSTATIC FIELDS (AN UNSOLVED PDE PROBLEM)
}

\author{
C. LO SURDO \\ Associazione EURATOM-ENEA sulla Fusione, Centro Ricerche Energia Frascati, \\ C.P. 65 - 00044 Frascati, Rome, Italy \\ (Received October 12, 1984)
}

ABSTRACT. A satisfactory theory of the Global MagnetoFluidoStatic (GMFS) Fields, where symmetric and non-symmetric configurations can be dealt with on the same footing, has not yet been developed. However the formulation of the Nowhere-Force-Free, Local-Global MFS problem about a given smooth isobaric toroidal surface $\mathscr{S}_{0}$ (actually, a degenerate initial-value problem) can be weakened so as to include certain generalızed solutions as formal power series in a "natural" transverse coordinate. It is reasonable to conjecture that these series converge, for sufficiently smooth data on $\mathscr{F}_{0}$, in the same function space which their coefficients belong to (in essence, a complete 1 inear space over the 2-torus).

KEY WORDS AND PHRASES. Local-Global Magnetofluidostatic Equilibria about an Isobaric Toroidal Surface, Degenerate Initial-Value Problem.

1980 MATHEMATICS SUBJECT CLASSIFICATION CODE : 76W05, 35Q

1. INTRODUCTION

Let $\Omega$ be a bounded, open, connected region $\subset \mathbb{R}^{3}$ with sufficiently smooth boundary $\partial \Omega$. One looks for a field $\left\{B=\left(B_{x}, B_{y}, B_{z}\right), P\right\}$ endowed with first derivatives in $\Omega$ and there fulfilling

$$
\nabla \times \mathrm{B} \times \mathrm{B}-\nabla \mathrm{P}=0, \quad \nabla \cdot \mathrm{B}=0,
$$

under the boundary condition $(B C)$

$$
\hat{\mathrm{v}} \times\left.\nabla \mathrm{P}\right|_{\partial \Omega}=0
$$

with $\hat{v} \doteq$ unit vector normal to $\partial \Omega$, for instance pointing outwards.

A local solution $\{B, P\}$ of eq. $\left(1.1_{1}\right)$ will be termed as a Magnetofluidostatic Field (MFSF), since $B$ and $P$ can be thought of as the magnetic induction and, respectively, the pressure which exist in a conducting fluid in static equilibrium under the Lorentz's force. A MFSF fulfilling BC $\left(1.1_{2}\right)$ for the given $\Omega$ will be called S2-Global MFS field ( $\Omega$-GMFSF). Any $\{B, P\}$ field with $B \equiv$ an harmonic vector (hence $\mathrm{P} \equiv$ const) in $\Omega$ is a $\Omega$-GMFSF which we shall term as "trivial". There are two obvious equivalence relations over the $\Omega$-GMFSF set $S_{\Omega}$. The first one, say $R_{1}$, is the equa- 
lity modulo a trivial field; the second one, $R_{2}$, is the homogeneity relation $\{B, P\} \sim$ $\sim\left\{k B, k^{2} P\right\}$ for any (real) constant $k$. So, one could confine himself to consider the quotient set $S_{\stackrel{\leftrightarrow}{\circ}} \doteq\left(S_{\Omega} / R_{1}\right) / R_{2}$ (or, if one likes, $\left.\left(S_{\Omega} / R_{2}\right) / R_{1}\right)$ in place of $S_{\Omega}$.

A special subset of $S_{\Omega}$ is that of the (non-trivial) $\Omega$-GMFSF's with $P=$ const in $\Omega$, the so-called "Force-Free" $(\Omega-G)$ fields. More particularly, there are force-free fields for which $\nabla \times B=\alpha B$ in $\Omega$ with $\alpha \equiv$ a real constant (the field "abnormality") in $\Omega$, or Trkal's fields (after a paper [1] of V. Trkal dated back to 1919). Trkal's fields can be easily constructed by solving a linear, 2nd-kind Fredholm equation (Lo Surdo [2]). Conversely, one could consider $\Omega$-GMFSF's for which $\nabla P \neq 0$ everywhere in $\Omega$ (possibly to be termed as "Nowhere Force-Free" fields).

Quite a natural, and still open, question (E) is that of determining the (non-trivial) elements of $S_{\hat{\Omega}}^{*}$ (if any). In this concern, the seemingly reasonable request $\{B, P\} \in C^{1}(\Omega)$ appears exceedingly strong, in the sense that, excluding trivial and Trkal's elements, $S_{\hat{\Omega}}^{\star}$ is then likely to be void for a generic $\Omega$ : in fact, only axiand/or plane-symmetric $C^{1}(\Omega) \Omega$-GMFSF's are presently known to exist (in particular requiring a similarly symmetric $\Omega$ ). So, one is led to allow a suitably enlarged solution space, also taking into consideration a wider acceptation of the $\Omega$-GMFSF concept. This should be done by a convenient weaker reformulation of eqs (1.1), possibly in the framework of more or less familiar ideas of modern non-linear functional analysis.

For instance, one could try to introduce some "test" Banach's space over $\Omega$, $\mathscr{T}(\Omega)$, a suitable (solution) linear space over $\Omega, L(\Omega) \supsetneqq \mathrm{C}^{1}(\Omega)$ and a non-linear mapping $\mathscr{U}: \mathrm{L}(\Omega) \rightarrow \mathscr{T}^{*}(\Omega)\left(\mathscr{T}^{*} \doteq\right.$ dual of $\left.\mathscr{T}\right)$, such that, with $\mathrm{X} \doteq\{\mathrm{B}, \mathrm{P}\}, \mathscr{M}(\mathrm{X})=0$ be "representative enough" of the original equations. Unfortunately, a program in the sense sketched above (say, problem $E^{*}$ ) appears a very difficult task. We note in particular that, unlike the familiar case of the stationary Navier-Stokes (NS) system without force, the lack of a corresponding elliptic term in eq. (1.1 1 ) (or, rather, in $\left.B \cdot \nabla B=\nabla\left(P+B^{2} / 2\right)\right)$ plays a deeply negative role in that it prevents the appropriate a priori estimates to exploit a fixed point theorem. On the other hand, a possible transition from that case to the present one (apart from the somewhat unusual $\mathrm{BC} \hat{\mathrm{v}} \times\left.\nabla\left(\mathrm{P} *+\mathrm{B}^{2} / 2\right)\right|_{\partial \Omega}=0$, with $\mathrm{P} \dot{\doteq}-\left(\mathrm{P}+\mathrm{B}^{2} / 2\right) \equiv$ NS pressure $)$, via singular-perturbation techniques, does not seem to have been ever attempted (see, among other sources, Lions [3], p. 396).

A variational formulation of the problem of constructing $\Omega$-GMFSF's under additional conditions to ensure uniqueness has been proposed (see e.g. Grad et al. [4], Kruskal et al. [5]) and extensively investigated by direct numerical methods (also with reference to a more general two-region configuration with $P=0$ in the second region and free interface, (Bauer et al. [6])). The success of these computations, which seems largely due to the rounding-off smoothing effects, can be reasonably interpreted as an indication of the existence of "generalized" (in some sense) solutions.

Somewhat from an opposite standpoint, the following statement holds true, being in turn a corollary of a powerful theorem of Arnold [7]. Assume $\{U, V\}$ to be an analytical solution to 


$$
\begin{aligned}
& \nabla \times(U \times V)=0, \\
& \nabla \cdot U=\nabla \cdot V=0
\end{aligned}
$$

in $\Omega$ with $B C\left(1.1_{2}\right)$ on $\pi \doteq \int_{x_{0}} d x \cdot U \times V, x_{c} \equiv a$ fixed point of $\bar{\Omega}$, under the "nowhere-force-free" constraint $U \times V \neq 0$ in $\bar{\Omega}$. Then it turns out that the surfaces $\pi=$ $=$ const $($ in $\bar{\Omega})$ are homeomorphic $(\tilde{\sim})$ to the 2 -torus $\mathrm{T}^{2}$. This also implies that $\bar{\Omega} \sim$ $\sim \mathrm{T}^{2} \times[0,1]$, i.e. that $\bar{\Omega}$ be a toroidal annulus foliated in toroidal $\left(\tilde{\sim} \mathrm{T}^{2}\right)$ surfaces between $\pi=\pi_{\mathrm{m}}$ and $\pi=\pi_{\mathrm{M}}, \pi_{\mathrm{m}}<\pi_{\mathrm{M}}$. We shall denote as $\mathrm{E}^{+}$the specialization of $\mathrm{E}$ (or, rather, of $\left.\mathrm{E}^{*}\right)$ to this case $" \bar{\Omega} \approx \mathrm{T}^{2} \times[0,1], \nabla \mathrm{P} \neq 0$ in $\overline{\Omega^{\prime}}$, which is also mostly important for the physical applications.

2. LOCAL-GLOBAL MFSF'S

Quite a natural local version of $\mathrm{E}^{+}$, say $\mathrm{E}_{\mathrm{L}}^{+}$, is that one obtains by looking for possible "Local-Global" MFSF's in a shell of sufficiently small measure about a given, smooth toroidal surface $\mathscr{f}_{0}$, where $\mathrm{P}=0$ (say). Although this more modest objective too gets into considerable difficulties (as expected), its analysis turns out of some help to enlighten the most critical aspects of the original problem.

First considering $E_{L}^{+}$from a classical point of view, we observe that it is certainly underdetermined if seen as an Initial Value (IV) problem, since the initial $B$, say $B_{0}$, is not prescribed. On the other hand, a surface $P=$ const (say $\mathscr{P}$ ) is characteristic of order 2 for eqs (1.1 $)$, implying a non-standard treatment of the IV problem. In fact i) two (differential) constraints must be fulfilled by the initial $\mathrm{B}_{0}$ on $\mathscr{H}_{\mathrm{o}}$. Assuming this compatible $\mathrm{B}_{\mathrm{o}}$ to exist and to have been fixed, the IV problem splits into two subproblems: ii) the "interior" subproblem of finding out the transverse (w.r.t. ' $f_{0}$ ) derivatives of two unknowns (such problem ii) turns out linear), and iij) the "exterior" problem of solving a system of four coupled, non-linear equations of the "evolution" type (with the transverse coordinate in place of time), i.e. one equating the transverse derivatives of the four unknowns to certain (after ii) has been solved) non-linear integral transforms of the unknowns themselves over the characteristic surface. As a matter of fact the root of all troubles resides in the linear problem $i i)$, which is likely to have no classical solutions.

To be more specific: as for $i$ ), one finds that

$$
\nabla \cdot \mathrm{B}_{\mathrm{O}}=0 \text {, }
$$

with $\nabla^{\prime} \times$ being the surface-curl operator. Condition (2.1) can be satisfied in infinitely many ways for a given $\mathscr{f}_{0}$. As for $\left.i i\right)$, one gets two coupled linear PDE's of type

$$
B^{\alpha} \partial_{\alpha}\left(\partial_{3} B^{\beta}\right)+\mathscr{A}_{\alpha}^{\beta} \partial_{3} B^{\alpha}+\mathscr{B}^{\beta}=0
$$

Here $\partial_{i} \equiv \partial / \partial \xi^{i}(i=1,2,3),\left(\xi^{1}, \xi^{2}\right)$ are (non-singular, smooth) coordinates all over $\mathscr{F}_{0}$, and $\xi^{3}$ is a transverse coordinate; say, the (sufficiently small) distance from $\mathscr{F}_{0}$, with sign, along the normal direction (to $\mathscr{S}_{0}$ ). $\mathscr{A}_{\alpha}^{\beta}$ and $\mathscr{B}^{\beta}$ are a surface 2nd-rank tensor and respectively a surface vector, both computable in terms of the "interior" functions ${ }^{\beta}$ once $\mathscr{f}_{\mathrm{O}}$ has been fixed. (Standard notations of the tensor calculus have been used, with greek indices ranging over $(1,2))$. 
The two remaining equations give $\partial_{3} B^{3}$, and respectively $\partial_{3} P$, in terms of interior tunctions and (as far as $\partial_{3} P$ is concerned) of $\partial_{3} B_{\beta}$. Should Eqs (2.2) in the $\partial_{3} B^{\beta^{\prime}}$ s have classical solutions, one would end up (after replacement of the latter into the expression for $\partial_{3} P$ ) with a system of type

$$
\begin{aligned}
& \partial_{3} B^{\beta}=\ldots \quad(\text { from eqs }(2.2)) \\
& \partial_{3} B^{3}=\ldots \\
& \partial_{3} P=\ldots
\end{aligned}
$$

with the dots denoting certain non-linear transforms of the $B^{\beta^{\prime}}$ s.

Being interested into a local solution, one could compute in a similar way the corresponding higher-order $\xi^{3}$-derivatives to get (formal) series in powers of $\xi^{3}$. A closer examination of problem ii) will show that such a (seemingly naif) resort is far from being a matter of convenience.

Before going on, however, we find it worth illustrating a neater alternative to Eqs $(2.1,2.2,2.3)$ based on the use of different unknowns. Let $\Gamma \equiv\left(\Gamma_{1}, \Gamma_{2}\right)$ be so-called Euler potentials of $U$ and respectively $V$ (see p. 3) according to:

$$
\begin{aligned}
& \mathrm{U}=\nabla \pi \times \nabla^{\prime} \Gamma_{1}, \\
& \mathrm{~V}=\nabla \pi \times \nabla^{\prime} \Gamma_{2},
\end{aligned}
$$

$\left(\nabla^{\prime} \doteq\right.$ surface gradient $)$, and let $\hat{\Gamma} \equiv\left\{\Gamma_{\alpha \beta}\right\}$ be the $2 \times 2$ matrix of coefficients $\Gamma_{\alpha \beta} \doteq$ $\doteq$ the increment of $\Gamma_{\alpha}$ along a cycle of class $\beta$ on the toroidal surface $\mathscr{f}$ (i.e. a (simple) cycle the long (say, $\beta=1$ ) or short $(\beta=2)$ way all around.P). Clearly, such $\Gamma_{\alpha \beta}$ are surface invariants. Then put:

$$
\begin{gathered}
\left(z^{1}, z^{2}\right) \equiv z \doteq \hat{\digamma}-1 \Gamma \\
z^{3} \doteq \int_{0} d \pi \operatorname{det} \hat{\Gamma} .
\end{gathered}
$$

It easily seen that $\operatorname{det} \hat{\imath} \neq 0$ follows from $U \times V \neq 0$. By definition, $z^{\alpha}$ increases by 1 along a cycle of class $(\alpha)$ and is a periodic function with zero average along a cycle of the other class. (Ultimately, $\left(Z^{1}, Z^{2}\right)$ are nothing but a convenient linear transform - to get "coordinate" cycles - of the so-called "flat" coordinate system on $\mathscr{f}$ corresponding to the completely integrable, non-singular vector system U,V (see for instance $[8], \mathrm{Ch} .9)$ ). Then one can show that the (conjectured to exist) smooth mapping $x \in \bar{\Omega} \mapsto\left(Z, Z^{3}\right) \in T^{2} \times\left[-Z_{m}^{3}, Z_{M}^{3}\right]$, with $\left(-Z_{m}^{3}, Z_{M}^{3}\right) \doteq \int_{0}^{\left(-\pi_{m}, \pi_{M}\right)} d \pi \operatorname{det} \hat{\Gamma}$, $-\pi_{\mathrm{m}}<0<\pi_{\mathrm{M}}$, ruled by the PD system

$$
\begin{gathered}
\frac{\partial\left(Z, Z^{3}\right)}{\partial(x)}=1, \\
\nabla \times\left(\nabla Z^{3} \times \nabla^{\prime} Z^{\alpha} \Gamma_{2 \alpha} / \operatorname{det} \hat{\Gamma}\right)=\nabla Z^{3} \times \nabla^{\prime} z^{\alpha} \Gamma_{1 \alpha} / \operatorname{det} \hat{\Gamma},
\end{gathered}
$$

(the second one representing the additional relationship $\nabla \times V=U$ ) is one-to-one. This justifies reformulating our original PD system as: 


$$
\begin{gathered}
\nabla P \cdot \nabla T_{1}^{\prime} \times \nabla^{\prime} \Gamma_{2}=1 \\
\nabla \times\left(\nabla P \times \nabla^{\prime} \Gamma_{2}\right)=\nabla P \times \nabla^{\prime} \Gamma_{1},
\end{gathered}
$$

by interchanging the roles of the independent and dependent variables; namely, we stipulate of looking for $x$ as a function of $\left(Z, Z^{3}\right)$ rather than for $\left(Z, Z^{3}\right)$ as a function of $x$ (eqs (2.6)). Such an "inverse mapping" version of the problem has important formal advatanges; namely, the independent domain becomes the canonical toric annulus $T^{2} \times\left[-Z_{m}^{3}, Z_{M}^{3}\right]$, the unknown $(x)$ is single-valued, and the magnetic lines $\mathrm{d} \times \mathrm{X}=0 \mathrm{map}$ onto straight lines of $\mathrm{T}^{2}$. The inverse PD system corresponding to system (2.6) is easily found to be:

$$
\begin{gathered}
\frac{\partial(\mathrm{x})}{\partial\left(\mathrm{Z}, \mathrm{Z}^{3}\right)}=1, \\
\left.2 \partial_{\beta \beta}\left(\partial_{3}\right]^{\mathrm{x}} \cdot \partial_{\alpha} \mathrm{x} \mathrm{T}_{2}{ }^{\alpha}\right)=\mathrm{T}_{1}{ }^{\alpha} \varepsilon_{\alpha \beta}, \\
\mathrm{T}_{2}^{\alpha} \partial_{1} \mathrm{x} \cdot \partial_{2}^{2} \mathrm{x}=0 .
\end{gathered}
$$

Here the derivatives are taken w.r.t. $\left.\left(Z^{1}, Z^{2}, Z^{3}\right), \perp\right\rfloor$ means antisymmetrization, $\mathrm{T}_{\gamma}^{\beta} \doteq \mathscr{E}^{\alpha \beta} \Gamma_{\gamma \alpha} /$ det $\hat{\Gamma}$ and $\mathscr{E}_{\alpha \beta}, \mathcal{E}{ }^{\alpha \beta}$ are the usual two-index antisymmetric capacity and density. (Note that $\mathrm{T}_{\gamma}^{\beta}$ only depends on $\mathrm{z}^{3}$ ).

Equation $\left(2.8_{3}\right)$ is a mere initial condition (add the $\partial_{2}$ of eq. $\left(2.8_{2}\right)$ for $\beta=2$ to the $\partial_{1}$ of eq. $\left(2.8_{2}\right)$ for $\left.\beta=1\right)$, mirroring the fact that only two scalar equations are actually independent in eq. $\left(2.7_{2}\right)$; its being fulfilled at $Z^{3}=0$ is the present version of problem $i$ ) (this should not be interpreted as an intrinsic constraint on $\mathscr{P}_{0}$, of course). One sees at once that a possible solution $x=x\left(Z, Z^{3}\right)$ would contain four arbitrary functions of one variable (the $T_{\alpha}^{\beta}\left(Z^{3}\right)$ 's) a circumstance that was not as well explicit in the previous formulation.

3. DISCUSSION

To see how problems of type $i i)$ and $i i i)$ emerge from eqs $\left(2.8_{1}, 2.8_{2}\right)$, we first introduce $g_{\alpha} \equiv g_{3 \alpha} \equiv \partial_{3} x \cdot \partial_{\alpha} x$ as auxiliary unknowns. Then ii) takes the form:

$$
\mathrm{T}_{2}^{\alpha} \partial_{\alpha} \mathrm{g}_{\beta}+\mathrm{b}_{\beta}^{\alpha} \mathrm{g}_{\alpha}=\mathrm{c}_{\beta}
$$

where

and

$$
\mathrm{b}_{\beta}^{\alpha} \doteq-2 \mathrm{~T}_{2}^{\gamma} \mathrm{p}^{\alpha \delta} \mathrm{m}_{\delta, \gamma \beta}
$$

$$
c_{\beta} \doteq 2 p^{\frac{1}{2}} \mathrm{~T}_{2}^{\alpha} \mathrm{q}_{\alpha \beta}-\mathrm{d}_{3} \mathrm{~T}_{2}^{\alpha} \mathrm{p}_{\alpha \beta}-\mathscr{E}_{\alpha \beta} \mathrm{T}_{1}^{\alpha}
$$

Here $p_{\alpha \beta}, q_{\alpha \beta}$ are the first and second fundamental tensor of the surface $z^{3}=$ const,

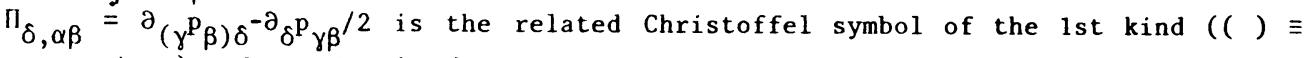
symmetrizer) and $\mathrm{p}=\operatorname{det}\left(\mathrm{p}_{\alpha \beta}\right)$.

System (3.1) is symmetric hyperbolic with the straight-line images of the magnetic lines, counted twice, as characteristic lines; if one likes, it is ordinary differential along these lines. It is intuitive that the general existence of a 
global, regular solution of (3.1) can be seriously questioned due to the topological structure of both the independent domain $\left(\mathrm{T}^{2}\right)$ and of the characteristic-line family on it. Here we meet the crucial point which makes the GMFSF theory so hard. A more specific, yet heuristic, discussion of the question runs as follows. First we re-write system (3.1) in matrix form for the compactness's sake:

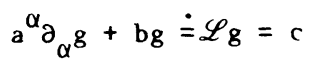

$\left(\mathrm{a}^{\alpha} \doteq \delta \mathrm{T}_{2}^{\alpha}, \delta \doteq\right.$ unit $2 \times 2$ matrix, etc). Assuming $\mathrm{T}_{2}{ }^{2} \neq 0\left(\left(\mathrm{~T}_{2}{ }^{1}\right)^{2}+\left(\mathrm{T}_{2}{ }^{2}\right)^{2}>0\right.$ in any case), then one can show that, under mild restrictions, the section $g\left(Z^{1}\right)$ along $Z^{2}=0$ of a $C^{1}$ solution of system ( $3.1 \mathrm{bis}$ ) (if any) must satisfy the linear finite-difference system:

$$
\stackrel{\circ}{g}\left(Z^{1}\right)-\zeta_{\rho}\left(Z^{1}\right) \stackrel{\circ}{g}\left(Z^{1}-\rho\right)=\sigma_{\rho}\left(Z^{1}\right) .
$$

Here $\rho \doteq \mathrm{T}_{2}{ }^{1} / \mathrm{T}_{2}{ }^{2}$, and $\zeta_{\rho}$ (a $2 \times 2$ matrix) and $\sigma_{\rho}$ (a 2-column) are integral transforms, both along the segment $\left[\left(Z^{1}-\rho, 0\right),\left(Z^{1}, 1\right)\right]$, of $b$ and, respectively, of $b$ and $c$, the latter being linear in $c$. (Of course, the solution $g$ would be then computed as sum of two linear integral transform of $\stackrel{\circ}{g}$ over $[0,1]$ and, respectively, of $c$ over tine strip $\left.[0,1] \times\left[0, Z^{2}\right]\right)$.

Curious as it may appear, no general existence theorem for system (3.2) and arbitrarily given real $\rho, \zeta_{\rho}, \sigma_{\rho}$ (and not even for a single equation of the same type) seems to be presently known. A relatively simple argument in this concern, anyhow, is as follows. Let us allow $\stackrel{\circ}{g}\left(Z^{1}\right)$ to be merely expandable as a trigonometrical series (which is much more permissive than be $C^{1}$, of course). Then it turns out that, however smooth $\zeta_{\rho}$ and $\sigma_{\rho}$ may be, the linear operator acting on $g\left(Z^{1}\right)$ is generally singular on a zero-measure, dense set $R$ of critical values of $\rho$, and that the null space associated to each of these values has dimension $\infty$. In other words, a "small-divisor"-wise phenomenon occurs; the problem being fredholmian, this means that infinitely many constraints between $\zeta_{\rho}$ and $\sigma_{\rho}$ must be fulfilled for almost all $\rho^{\prime}$ s for a trigonometrically expandable solution to exist (not to mention the convergence requirement). In conclusion it seems quite unplausible that the existence of a classical solution all over a $Z^{3}$ - interval about $Z^{3}=0$ might follow from the peculiar nature of $a^{\alpha}, b, c$. One is thus forced to regard system (3.1) as a genuine PD system, looking for generalized (in principle, distributional) solutions. This also justifies a previous remark. Indeed, assuming system (3.1) to have been solved - in some appropriate sense - at $Z^{3}=0$,

$$
\partial_{3} x=p^{\alpha \beta} g_{\beta} \partial_{\alpha} x+p^{-1} \partial_{1} x \times \partial_{2} x
$$

will turn out generally non-smooth there, due to the presence of the $g_{\beta}$ 's on the RHS. In other words, one would get non-smooth neighbouring "isobaric" surfaces $x=$ $=x\left(Z, Z^{3}=\right.$ const $\left.\neq 0\right)$ to 1 st order in $Z^{3}$. This implies that $\mathscr{L}$ need not to be even defined over surfaces close to $Z^{3}=0$. The working with a power series expansion w.r.t. $Z^{3}$ avoids this basic difficulty, though it opens new, but presumably more accessible, questions. Of course one would (hopefully) end up with an asymptotic solution $\left(Z^{3} \rightarrow 0\right)$ in this case, unless the related series can be proven to converge, possibly in the same space which their coefficients belong to, for some non-zero $Z^{3}$. 
Another way out of the same trouble would be the resorting to a possible approximate, but smooth, solution of eqs (3.1). (In passing, we note also that eq. (3.2), with the $g_{\beta}$ 's eliminated via eqs (3.1), is the present version of problem iii)).

With reference to the power series expansion approach, the hierarchy of $Z^{3}$-derivatives of eqs (3.1) has the form:

$$
\begin{gathered}
\left.\left(\left.\mathscr{L}_{\mathrm{g}-\mathrm{c})}\right|_{\mathrm{o}} \doteq\left(\mathscr{L}_{\mathrm{g}}{ }_{\mathrm{o}}\right)-\mathrm{c}^{(\mathrm{o})}\right)\right|_{\mathrm{o}}=0 \\
\left.\left.\left(\mathscr{L} \mathrm{g}_{3} \mathrm{~g}+\mathrm{\partial}_{3} \mathscr{L}_{\mathrm{g}}-\partial_{3} \mathrm{c}\right)\right|_{\mathrm{o}} \doteq\left(\mathscr{L}_{\mathrm{g}}{ }^{(1)}-\mathrm{c}^{\left({ }_{1}\right)}\right)\right|_{\mathrm{o}}=0 \\
\left.\left.\left(\mathscr{L} \partial \frac{2}{3} \mathrm{~g}+\ldots\right)\right|_{\mathrm{o}} \doteq\left(\mathscr{L}_{\mathrm{g}}{ }^{\left({ }_{2}\right)}-\mathrm{c}^{\left({ }_{2}\right)}\right)\right|_{\mathrm{o}}=0,
\end{gathered}
$$

and so on.

Symmetric hyperbolic systems over $T^{2}-11$ ke each of the above systems $\left(3.1\right.$ ter $\left.{ }_{m \geq 0}\right)$ - (or even over $T^{n}$ ) have been intensively studied, and it would be easy to indicate sufficient conditions for a solution in such and such function space to exist. For instance considering solutions which belong to the Hilbert space $\mathscr{H}_{2} \doteq \mathrm{L}_{2}\left(\mathrm{~T}^{2}\right) \times \mathrm{L}_{2}\left(\mathrm{~T}^{2}\right)$, the weak version of system (3.1ter) would be (neglecting the $I_{0}$ for the brevity's sake):

$$
\left(\mathrm{g}^{(\mathrm{m})}, \mathscr{L}^{+} \phi\right)=\left(\mathrm{c}^{(\mathrm{m})}, \phi\right), \quad(\mathrm{m}=0,1,2 \ldots)
$$

with $\mathscr{L}^{+}$being the formal adjoint of $\mathscr{L}, \mathscr{L}^{+} \doteq-\mathrm{a}^{\alpha} \partial_{\alpha}+\mathrm{b}^{+}, \mathrm{b}^{+} \doteq$ adjoint of $\mathrm{b}$, for al1 test 2-columns $\phi \in \mathrm{C}^{1}\left(\mathrm{~T}^{2}\right) \times \mathrm{C}^{1}\left(\mathrm{~T}^{2}\right)$. This obviously presupposes that the $\mathrm{c}^{(\mathrm{m})}{ }^{\prime} \mathbf{s}$ all belong to $\mathscr{H}_{2}$, which can be proved true for sufficiently smooth $x(Z, 0)$ and (say) analytical $a^{\alpha}\left(Z^{3}\right)$ at $Z^{3}=0$ (see [9]). Then a solution $\mathrm{g}^{(\mathrm{m})}$ exists if and only if $\mathscr{L}^{+} \phi=0 \Rightarrow\left(\mathrm{c}^{(\mathrm{m})}, \phi\right)=0$ for all $\phi^{\prime} \mathrm{s}$; in particular, for $\mathscr{L}^{+}$bounded below. Another interesting (sufficient) condition is when $\bar{b} \equiv b^{+} b^{+}$be definite (positive or negative) over $\nVdash_{2}$, i.e. when [10]

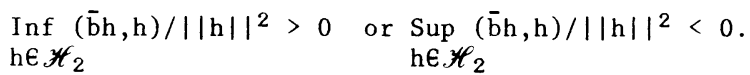

This makes sense for sufficiently smooth $\bar{b}$, so that $h \in \mathscr{H}_{2} \Rightarrow \bar{b} h € \mathscr{H}_{2}$. What remains hard is the ascertaining whether some of the above conditions might be validated by convenient initial data, without falling in the usual symmetries.

This problem will not be pursued here. However, a remark is in order in this respect. Since $C^{1} \Omega$-GMFSF's are known to exist in the symmetric configurations mentioned on p. 2, one expects that system (3.1) be classically solvable in those cases. This has been proved in [9, App. 3]. Instead, the proof of the (plausible) converse statement - that the classical solvability over a $Z^{3}$-interval automatically singles out one of the above symmetries - does not seem an easy task.

4. CONCLUDING REMARKS

Coming to a close of this note, we attempt to summarize the whole of the above considerations as follows. The existence problem of concern: 
1) does not allows a classical approach, so that a "weak" treatment, in a sense or in another, is a need with no way out. Quoting from H. Grad [11], here "we are presented with an unusual situation in PDE's, where weak solutions are not adopted as a mathematical convenience, but at the insistence of the equations" (the only word we do not agree with in the above is "unusual");

ii) seems to require unconventional ideas, even if tentatively tackled along modern functionalistic lines;

ii) is still unexplored as a singular-perturbation problem, via standard elliptic-regularization techniques;

$1 \mathrm{v})$ is an important and still open mathematical question, on which nothing really decisive has been published so far.

v) is of strong relevance to magnetofluidostatic, and in particular, to the statics (or to the dynamics, as long as inertial and viscosity effects are neglected in the momentum balance) of truly 3-dimensional, closed-type fusion devices (for instance, ohmicly heated Stellarators).

Lacking an effective, direct route to the solution of $E^{*}$, we have turned on the presumably more tractable, yet still significant, local-global problem (E ${ }^{+}$), showing that the main difficulties are tied to the inversion of a linear, PD symmetric hyperbolic $(2 \times 2)$ operator acting on a (1inear) space of two-fold periodic 2-columns; for instance, on $\mathscr{H}_{2}\left(\mathrm{~T}^{2}\right)$. Should this problem be solved in a sense or in another, the construction of a formal (or perhaps convergent, in the relevant topology) power series solution, about the inttial isobaric surface, would be quite conceivable.

\section{REFERENCES}

1. TRKAL, V.: Paznamka k hydrodynamice va z kych tekutin, Casopis pro Pestovani Mathematiki a Fisiky (Praha) 48 (1919) 302-311.

2. LO SURDO, C.: "Pseudostatic Resistive Hydromagnetic Equilibria in a Toroid", J. Applied Math. and Physics (ZAMP), 30 (1979) 647-653.

3. LIONS, J.L.: "Perturbation Singulieres etc." Lecture Notes in Mathem. $n^{\circ} 323$, Springer, Berlin (1973).

4. GRAD, H., RUBIN, H.: "Hydromagnetic Equilibria and Force-Free Fields", 2nd Int. Conf. Peac. Uses of Atom. Energy, 31 (1958, Geneva), P. 386, 190-197.

5. KRUSKAL, M.D., KULSRUD, M.R.: "Equilıbria of a Magnetically Confined Plasma in a Toroid" Phys. Fluids, 1, (1958) 265-274.

6. BAUER F., et al.: "A Computational Method in Plasma Physics" Springer, Berlin (1978).

7. ARNOLD, V.: "Sur la topologie des écoulements stationnaires des fluids parfaits" C.R. Acad. Sci. Paris, 261, (1965) 17-20.

8. HERMANN, R., "Differential Geometry and the Calculus of Variations", Acad. Press, New York (1968).

9. LO SURDO, C.: "On the Local Global Magnetofluidostatic Problem", Report CNEN $81.45 / \mathrm{p}$, Associazione EURATOM-CNEN sulla Fusione (1981).

10. FRIEDRICHS, K.0.: "Symmetric Positive Linear Differential Equations" Comm. Pure Applied Mathemat. 11 (1958) 333-418.

11. GRAD, H.:"Problems in Magnetostatic Equilibrium", in "Intern. Colloquium on MHD", Lille (1969), and Int. Rep. MF-62, AFOSR 70-1134 TR, Courant Institute of Math. Sciences, MFD Division (1970) 


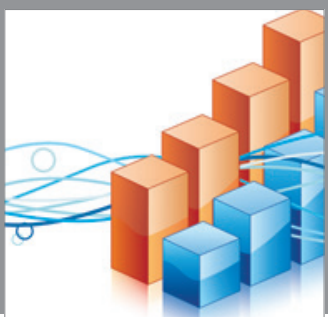

Advances in

Operations Research

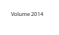

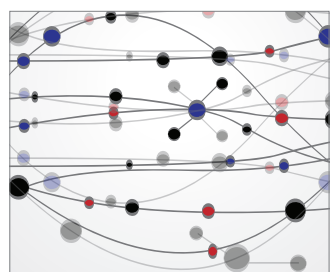

\section{The Scientific} World Journal
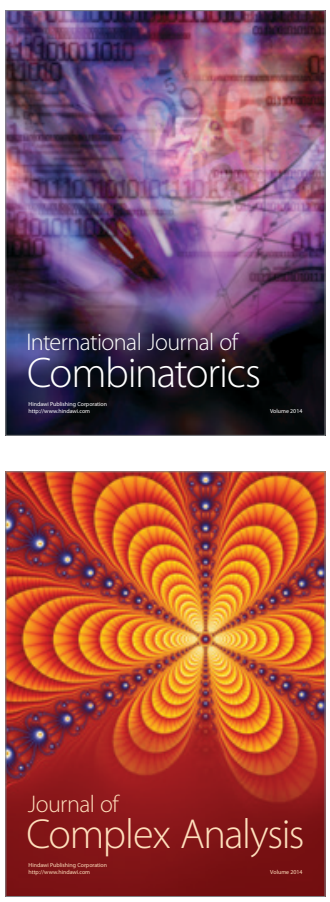

International Journal of

Mathematics and

Mathematical

Sciences
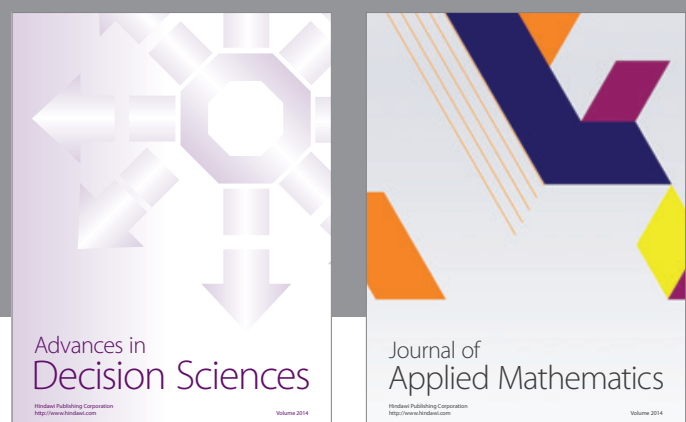

Journal of

Applied Mathematics
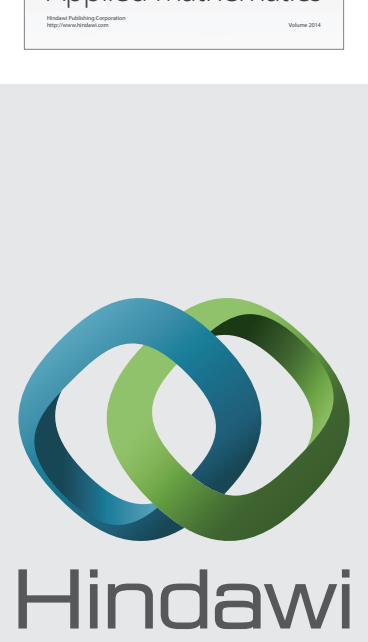

Submit your manuscripts at http://www.hindawi.com
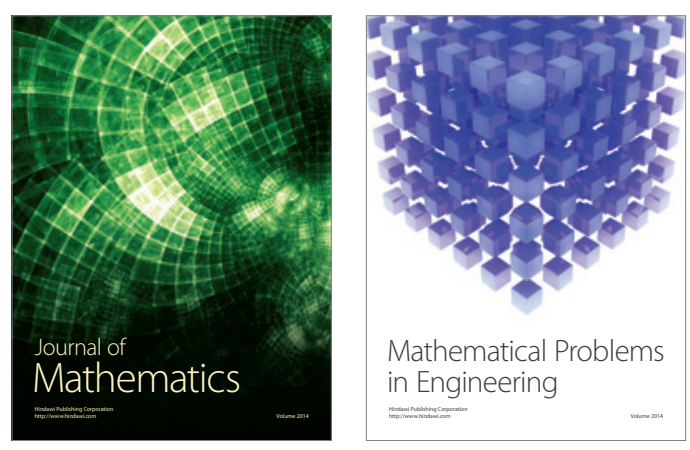

Mathematical Problems in Engineering
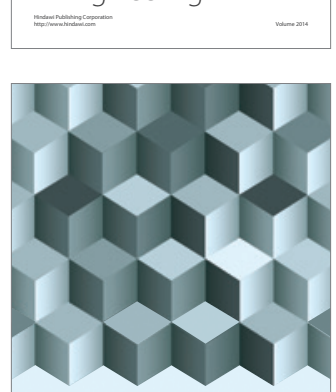

Journal of

Function Spaces
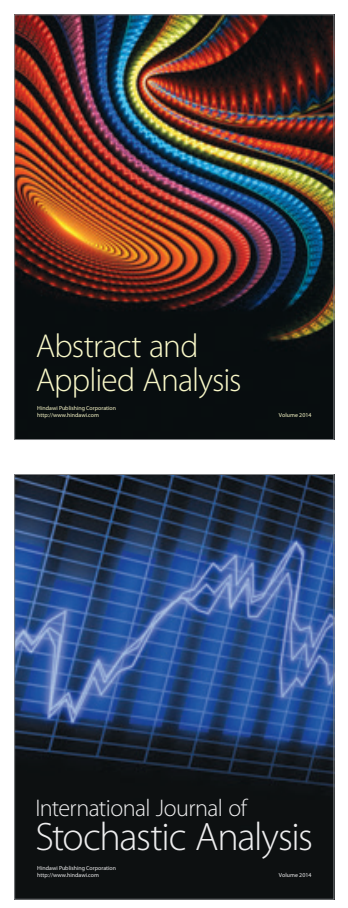

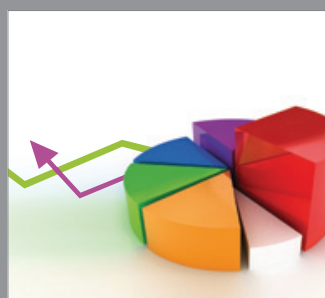

ournal of

Probability and Statistics

Promensencen
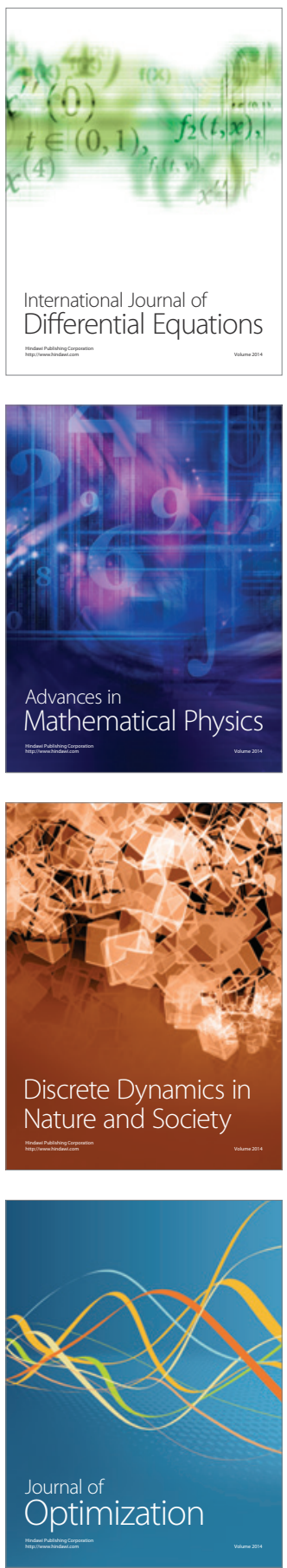